\title{
Rayleigh's, Stoneley's, and Scholte's Interface Waves in Elastic Models Using a Boundary Element Method
}

\author{
Esteban Flores-Mendez, ${ }^{1}$ Manuel Carbajal-Romero, ${ }^{2}$ \\ Norberto Flores-Guzmán, ${ }^{3}$ Ricardo Sánchez-Martínez, ${ }^{2}$ \\ and Alejandro Rodríguez-Castellanos ${ }^{4}$ \\ ${ }^{1}$ Sección de Estudios de Posgrado e Investigación, ESIA Zacatenco, Instituto Politécnico Nacional, \\ Avenida Instituto Politécnico Nacional s/n, Lindavista, Del. Gustavo A. Madero, 07320 México, \\ DF, Mexico \\ 2 Sección de Estudios de Posgrado e Investigación, ESIME Azcapotzalco, Instituto Politécnico Nacional, \\ Avenida de las Granjas 682, Sta. Catarina, Del. Azcapotzalco, 02250 México, DF, Mexico \\ ${ }^{3}$ Ciencias de la computación, Centro de Investigación en Matemáticas, Callejón Jalisco s/n, \\ Mineral de Valenciana, 36240 Guanajuato, GTO, Mexico \\ ${ }^{4}$ Programa de Investigación de Geofísica de Exploración y Explotación, Instituto Mexicano del Petróleo, \\ Eje Central Lázaro Cárdenas 152, Gustavo A. Madero, 07730 México, DF, Mexico
}

Correspondence should be addressed to Esteban Flores-Mendez, flores-mendez.e@hotmail.com

Received 15 September 2011; Revised 5 December 2011; Accepted 7 December 2011

Academic Editor: Srinivasan Natesan

Copyright (C) 2012 Esteban Flores-Mendez et al. This is an open access article distributed under the Creative Commons Attribution License, which permits unrestricted use, distribution, and reproduction in any medium, provided the original work is properly cited.

This work is focused on studying interface waves for three canonical models, that is, interfaces formed by vacuum-solid, solid-solid, and liquid-solid. These interfaces excited by dynamic loads cause the emergence of Rayleigh's, Stoneley's, and Scholte's waves, respectively. To perform the study, the indirect boundary element method is used, which has proved to be a powerful tool for numerical modeling of problems in elastodynamics. In essence, the method expresses the diffracted wave field of stresses, pressures, and displacements by a boundary integral, also known as single-layer representation, whose shape can be regarded as a Fredholm's integral representation of second kind and zero order. This representation can be considered as an exemplification of Huygens' principle, which is equivalent to Somigliana's representation theorem. Results in frequency domain for the three types of interfaces are presented; then, using the fourier discrete transform, we derive the results in time domain, where the emergence of interface waves is highlighted.

\section{Introduction}

The study of interface waves has always attracted the interest of the scientific community because of the importance and complexity of the waves that propagate in such interfaces. 
For example, Rayleigh's waves are one of the three types of interface waves, which travel in vacuum-solid surfaces. In isotropic solids the particle motion is elliptical and retrograde, for shallow depths, with respect to the direction of propagation (Rayleigh, [1]). Today, many engineering and seismology studies (e.g., [2-5]) are focused on understanding Rayleigh's waves. Recent research concerning Rayleigh's waves is also carried out on nondestructive testing for detecting defects.

Stoneley's waves occur at the interface between two solids [6]. The higher energy, as well as Rayleigh's waves, is present in the interface and shows an exponential decay away from the interface. Important applications around this type of interface waves can be found in [7-10].

Scholte's waves are presented at the interface of fluid-solid media [11-13]. Similarly, most of the energy in this type of wave is presented in the interface and decays exponentially into the solid medium and fluid one. Some applications, mainly applied to seabed, can be seen in $[8,14-17]$.

To model realistic problems and complex geometries, numerical methods are a good option. Methods like finite element [18], finite difference [19], boundary element [20, 21], spectral and pseudospectral elements [22-24] have been extensively used.

Particularly, the boundary element method (BEM) has been useful to deal with interface problems. For instance, based on the BEM, a coupled model was developed to investigate the dynamic interaction between an offshore pile, a porous seabed, and seawater when subjected to the pseudo-Stoneley wave along the seabed and the seawater interface [25]. They found that the maximum pore pressure of the seabed usually occurs at the region near the interfaces between the seabed and the seawater.

Numerical modeling to simulate the propagation of acoustic and elastic waves generated by a borehole source embedded in a layered medium was formulated in terms of the boundary element technique, where Green's functions were calculated by the discrete wavenumber method. Results display Stoneley's wave reflections at the bed boundaries and show the importance of the diffraction that takes place where the borehole wall intersects the layer interfaces [26].

Characterization of surface cracks using Rayleigh's wave excitations was dealt by an indirect boundary element method. The variations of spectral ratios between the transmitted and incident waves were studied as a function of the crack depth. They were used to design an efficient procedure for the determination of crack depths [27].

BEM formulations have been also used to study scattering of Rayleigh's wave by cavities [28]. Moreover, BEM methods were developed to study reflection and transmission of Rayleigh's surface waves by a juncture normal to free surface, between identical or different materials [29].

Propagation of Scholte's waves in a water-filled borehole in an anisotropic solid by a time-domain boundary element method was studied in [30], where detailed arrival identification and interpretation of acoustic and elastic waves propagating along the fluid-solid interfaces were pointed out.

In this paper, a numerical method known as the indirect boundary element method (IBEM) is used to study, in frequency and time domain, the behavior of three canonical models of interface giving rise to the emergence of Rayleigh's, Stoneley's, and Scholte's waves. To validate the equations used here, we included in The appendix, a comparison between the IBEM and discrete wave number (DWN), for the case of a fluid-solid interface, where an initial pressure is applied in the fluid using a Hankel's function of second kind and zero order. 


\section{Indirect Boundary Element Method Formulation}

For the three interface models studied in this work, the resulting state of tractions, displacements, and pressures at any point of the models can be expressed as the sum of an incident field and a diffracted one. All interface models, vacuum-solid, solid-solid, fluid-solid, are considered as the union of two half spaces or regions. The source generates, in the region where it acts, an incident field of pressures and displacements for the fluid or an incident field of tractions and displacement for solids. In the region where the source is not applied, only diffracted fields are expected.

For comparison and validation, the results obtained by this numerical technique are compared with respect to those obtained by the DWN. This comparison was performed for the case of a fluid-solid interface using a source in the fluid, which was represented by a Hankel's function of second kind and zero order; this validation is detailed in The appendix. However, for the purpose of making comparisons between the three interface models, the source is applied in the solid $S^{1}$, as shown in Figure 1.

Therefore, for each model, the total field for the $S^{1}$ region can be expressed as $t^{S^{1}}(\mathbf{x})=$

$t^{o S^{1}}(\mathbf{x})+t^{d^{S^{1}}}(\mathbf{x})$ for tractions, and $u^{S^{1}}(\mathbf{x})=u^{o S^{1}}(\mathbf{x})+u^{d^{S^{1}}}(\mathbf{x})$ for displacements. The regions $S^{2}$ and $F$ will only have diffracted wave fields, because no sources are applied in such regions. The superindexes $o$ and $d$ stand for incident and diffracted wave field, respectively.

\section{Integral Representations of Diffracted Fields}

The diffracted wave field of displacements and tractions for the elastic solid $S^{1}$ can be expressed by means of

$$
\begin{aligned}
& u_{i}^{d^{S^{1}}}(\mathbf{x})=\int_{\partial S^{1}} G_{i j}^{S^{1}}(\mathbf{x}, \xi) \phi_{j}^{S^{1}}(\xi) d S_{\xi}, \\
& t_{i}^{d^{S^{1}}}(\mathbf{x})=c_{1} \phi_{i}^{S^{1}}(\mathbf{x})+\int_{\partial S^{1}} T_{i j}^{S^{1}}(\mathbf{x}, \xi) \phi_{j}^{S^{1}}(\xi) d S_{\xi},
\end{aligned}
$$

where $u_{i}(\mathbf{x})$ represents the $i$ th component of the displacement at point $\mathbf{x}, G_{i j}(\mathbf{x} ; \xi)$ represents Green's function, which is the displacement produced in the direction $i$ at $\mathbf{x}$ due to the application of a unit force in direction $j$ at point $\xi$ and $\phi_{j}(\xi)$ is the force density in direction $j$ at point $\xi$. This integral representation can be obtained from Somigliana's identity [4]. $t_{i}(\mathbf{x})$ is the $i$ th component of traction, $c_{1}=0.5$, if $\mathbf{x}$ tends to the boundary $S$ "from inside" the region, $c_{1}=-0.5$ if $\mathbf{x}$ tends to $S$ "from outside" the region, and $c_{1}=0$ if $\mathbf{x}$ is not at $S . T_{i j}(\mathbf{x} ; \xi)$ is the traction Green's function, that is, the traction in the direction $i$ at a point $\mathbf{x}$, associated to the unit vector $n_{i}(\mathbf{x})$, due to the application of a unit force in the direction $j$ at $\xi$ on $S$. The 2D Green functions for unbounded spaces can be obtained in $[5,31]$. Diffracted wave field for the region $S^{2}$ has similar form to (3.1).

For the region $F$ (fluid in Figure 1(c)), the diffracted fields for displacements and pressures are written as

$$
\begin{gathered}
u_{n}^{d^{F}}(\mathbf{x})=c_{2} \Psi(\mathbf{x})+\frac{1}{\rho_{F} \omega^{2}} \int_{\partial F} \frac{\partial G^{F}(\mathbf{x}, \xi) \Psi(\xi) d S_{\xi}}{\partial n}, \\
p^{d^{F}}(\mathbf{x})=\int_{\partial F} G^{F}(\mathbf{x}, \xi) \Psi(\xi) d S_{\xi}
\end{gathered}
$$




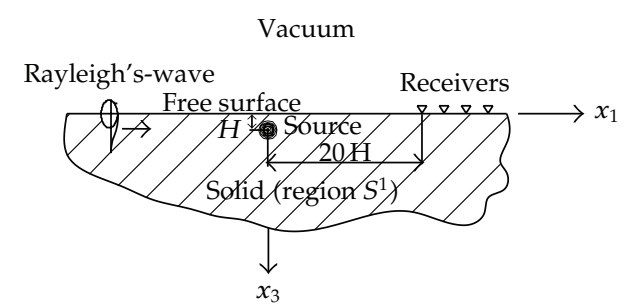

(a)
Solid (region $\left.S^{2}\right)$

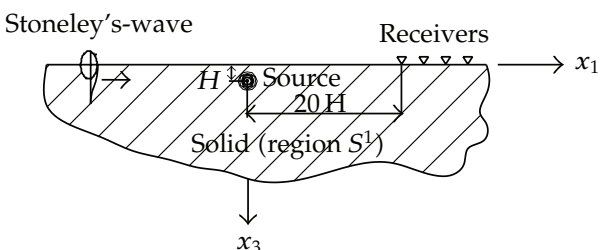

(b)

Fluid (region $F$ )

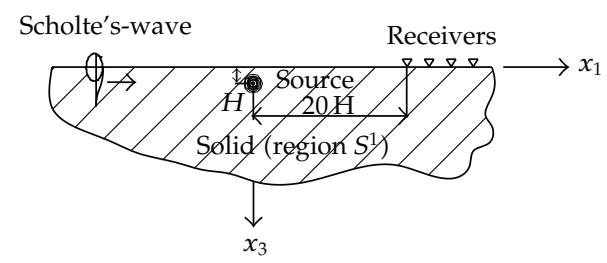

(c)

Figure 1: Canonical interface models to be solved by means of the indirect boundary element method. (a) Vacuum-solid interface, (b) solid-solid interface, and (c) fluid-solid interface.

where

$$
G^{F}(\mathbf{x}, \xi)=\frac{\rho \omega^{2}}{4 i} H_{0}^{(2)}\left(\frac{\omega r}{c^{F}}\right)
$$

$\Psi(\cdot)$ represents the force density for the fluid, $G^{F}(\cdot)$ is the Green function for the fluid, and $c_{2}$ defines the region orientation and can assume a value of $-0.5,0$, or 0.5 (see explanation for $c_{1}$, given above). $\rho$ is the mass density, and $\omega$ represents the circular frequency.

\subsection{Boundary Conditions}

Boundary conditions for each of the models presented in Figure 1 are set as follows:

Vacuum-solid interface

$$
t_{i}^{S^{1}}(\mathbf{x})=0, \quad \forall \mathbf{x} \in \partial S^{1}
$$

Solid-solid interface

$$
\begin{aligned}
& t_{i}^{S^{1}}(\mathbf{x})=t_{i}^{S^{2}}(\mathbf{x}), \quad \forall \mathbf{x} \in \partial S^{1}, \partial S^{2}, \\
& u_{i}^{S^{1}}(\mathbf{x})=u_{i}^{S^{2}}(\mathbf{x}), \quad \forall \mathbf{x} \in \partial S^{1}, \partial S^{2} .
\end{aligned}
$$


Fluid-solid interface

$$
\begin{gathered}
u_{3}^{F}(\mathbf{x})=u_{3}^{S^{1}}(\mathbf{x}), \quad \forall \mathbf{x} \in \partial S^{1}, \partial F \\
t_{1}^{S^{1}}(\mathbf{x})=0, \quad \forall \mathbf{x} \in \partial S^{1}, \\
t_{3}^{S^{1}}(\mathbf{x})=-p^{F}(\mathbf{x}), \quad \forall \mathbf{x} \in \partial S^{1}, \partial F .
\end{gathered}
$$

Equation (3.4) represents the traction state on a free surface, which promotes the emergence of Rayleigh's waves. Boundary conditions (3.5) ensure continuity between two materials with different mechanical properties and exhibit the existence of Stoneley's waves. Finally, (3.6) are the appropriate boundary conditions between an acoustic medium and an elastic solid one.

\section{Discretization Scheme}

For purposes of exemplification, the discretization procedure for the equations corresponding to the interface of Figure 1(b) is illustrated, such interface is related to the emergence and propagation of Stoneley's waves, which exist in the interface between two elastic solids. Then, from the equation of continuity (3.5), it can be said that the traction and displacement states may be expressed, respectively, as

$$
\begin{gathered}
t_{i}^{S^{S^{1}}}(\mathbf{x})+t_{i}^{d^{S^{1}}}(\mathbf{x})=t_{i}^{d^{S^{2}}}(\mathbf{x}), \quad \forall \mathbf{x} \in \partial S^{1}, \partial S^{2}, \\
u_{i}^{o^{S^{1}}}(\mathbf{x})+u_{i}^{d^{S^{1}}}(\mathbf{x})=u_{i}^{d^{S^{2}}}(\mathbf{x}), \quad \forall \mathbf{x} \in \partial S^{1}, \partial S^{2} .
\end{gathered}
$$

Reordering these last two equations, one has

$$
\begin{gathered}
t_{i}^{d^{S^{1}}}(\mathbf{x})-t_{i}^{d^{S^{2}}}(\mathbf{x})=-t_{i}^{o^{S^{1}}}(\mathbf{x}), \quad \forall \mathbf{x} \in \partial S^{1}, \partial S^{2}, \\
u_{i}^{d^{S^{1}}}(\mathbf{x})-u_{i}^{d^{S^{2}}}(\mathbf{x})=-u_{i}^{S^{S^{1}}}(\mathbf{x}), \quad \forall \mathbf{x} \in \partial S^{1}, \partial S^{2} .
\end{gathered}
$$

According to the integral representations (3.1), (4.3) can be written as

$$
\begin{aligned}
c_{1} \phi_{i}^{S^{1}}(\mathbf{x})+ & \int_{\partial S^{1}} T_{i j}^{S^{1}}(\mathbf{x}, \xi) \phi_{j}^{S^{1}}(\xi) d S_{\xi}-c_{2} \phi_{i}^{S^{2}}(\mathbf{x})-\int_{\partial S^{2}} T_{i j}^{S^{2}}(\mathbf{x}, \xi) \phi_{j}^{S^{2}}(\xi) d S_{\xi}=-t_{i}^{S^{1}}, \quad \forall \mathbf{x} \in \partial S^{1}, \partial S^{2}, \\
& \int_{\partial S^{1}} G_{i j}^{S^{1}}(\mathbf{x}, \xi) \phi_{j}^{S^{1}}(\xi) d S_{\xi}-\int_{\partial S^{2}} G_{i j}^{S^{2}}(\mathbf{x}, \xi) \phi_{j}^{S^{2}}(\xi) d S_{\xi}=-u_{i}^{S^{1}}, \quad \forall \mathbf{x} \in \partial S^{1}, \partial S^{2},
\end{aligned}
$$

where $t_{i}^{S^{s^{1}}}$ and $u_{i}^{o^{S^{1}}}$ represent, respectively, the stress and displacement wave fields produced by the source, both applied in the region $S^{1}$. 


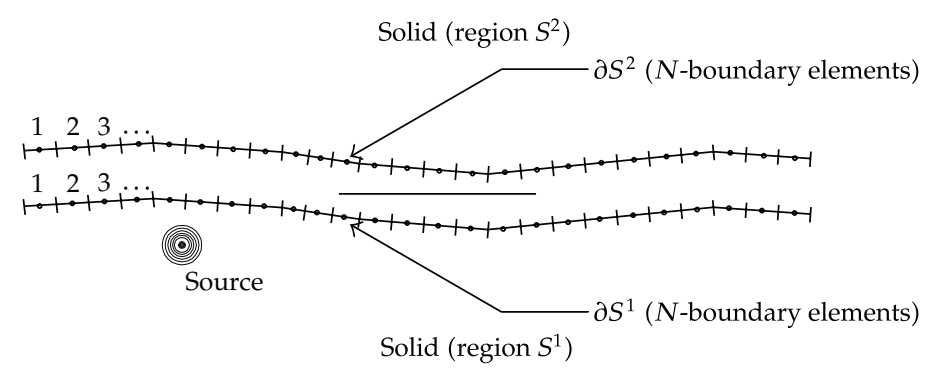

Figure 2: Boundary element mesh for Stoneley's problem.

In general, the interface between two solids may be discretized according to Figure 2.

If we assume that the force densities $\phi_{i}(\mathbf{x})$ are constant in each boundary element that forms the surfaces of the regions $S^{1}$ and $S^{2}$ and the Gaussian integration is performed (or analytical integration where Green's function is singular), then (4.4) can be rewritten as

$$
\begin{aligned}
& \sum_{n=1}^{N} \phi_{j}^{S^{1}}\left(\xi_{n}\right) t_{i j}^{S^{1}}\left(\mathbf{x}_{l}, \xi_{n}\right)-\sum_{n=1}^{N} \phi_{j}^{S^{2}}\left(\xi_{n}\right) t_{i j}^{S^{2}}\left(\mathbf{x}_{l}, \xi_{n}\right)=-t_{i}^{o^{S^{1}}}\left(\mathbf{x}_{l}\right), \quad l=1, N \\
& \sum_{n=1}^{N} \phi_{j}^{S^{1}}\left(\xi_{n}\right) u_{i j}^{S^{1}}\left(\mathbf{x}_{l}, \xi_{n}\right)-\sum_{n=1}^{N} \phi_{j}^{S^{2}}\left(\xi_{n}\right) u_{i j}^{S^{2}}\left(\mathbf{x}_{l}, \xi_{n}\right)=-u_{i}^{O^{S^{1}}}\left(\mathbf{x}_{l}\right), \quad l=1, N
\end{aligned}
$$

where

$$
\begin{aligned}
& t_{i j}\left(\mathbf{x}_{l}, \xi_{n}\right)=c_{1} \delta_{i j} \delta_{l n}+\int_{\Delta S_{n}} T_{i j}\left(\mathbf{x}_{l}, \xi_{n}\right) d S_{\xi}, \\
& g_{i j}\left(\mathbf{x}_{l}, \xi_{n}\right)=\int_{\Delta S_{n}} G_{i j}\left(\mathbf{x}_{l}, \xi_{n}\right) d S_{\xi},
\end{aligned}
$$

where $\delta_{i j}$ represents the Kronecker delta and $\Delta S_{n}$ is the length of each boundary element.

Equations (4.5) represent the system of Fredholm's integral equations to be solved. Once the unknowns are found, it is possible to determine the state of tractions and displacement at any point within the regions $S^{1}$ and $S^{2}$ using (3.1), plus the incident wave field.

For the other types of interface, it is possible to follow the same discretization scheme, applying the corresponding boundary conditions. Our integral representations can be used to handle nonflat interfaces, which is the subject of our current research.

\section{Numerical Examples}

For validation purposes we refer the reader to the appendix. There, results achieved by the IBEM are compared with those obtained by the DWN method, for the case of fluid-solid interfaces. Good agreement is seen between both methods.

In this section, numerical simulations for the three canonical interface models are developed. Flat interfaces are considered for the three models. The material properties used for the analysis are shown in Table 1; these material properties were consulted from [32-34]. $\alpha$ and 
Table 1: Material properties used for numerical simulations.

\begin{tabular}{|c|c|c|c|c|c|c|c|c|c|c|c|c|}
\hline \multirow{2}{*}{ Interface model } & \multicolumn{6}{|c|}{ Materials 1} & \multicolumn{6}{|c|}{ Materials 2} \\
\hline & $\alpha$ & $\beta$ & $\rho$ & $\alpha$ & $\beta$ & $\rho$ & $\alpha$ & $\beta$ & $\rho$ & $\alpha$ & $\beta$ & $\rho$ \\
\hline \multirow{2}{*}{ Vacuum-solid } & \multicolumn{6}{|c|}{ Solid $\left(S^{1}\right)$} & & & & \multicolumn{3}{|c|}{ Solid $\left(S^{1}\right)$} \\
\hline & & & & 2670 & 1090 & 2200 & & & & 4810 & 2195 & 2500 \\
\hline \multirow{2}{*}{ Solid-solid } & \multicolumn{3}{|c|}{ Solid $\left(S^{1}\right)$} & \multicolumn{3}{|c|}{ Solid $\left(S^{2}\right)$} & \multicolumn{3}{|c|}{ Solid $\left(S^{1}\right)$} & \multicolumn{3}{|c|}{ Solid $\left(S^{2}\right)$} \\
\hline & 2670 & 1090 & 2200 & 4810 & 2195 & 2500 & 4810 & 2195 & 2500 & 2670 & 1090 & 2200 \\
\hline \multirow[t]{2}{*}{ Fluid-solid } & \multicolumn{3}{|c|}{ Fluid $(F)$} & \multicolumn{3}{|c|}{ Solid $\left(S^{1}\right)$} & \multicolumn{3}{|c|}{ Fluid $(F)$} & \multicolumn{3}{|c|}{ Solid $\left(S^{1}\right)$} \\
\hline & 1501 & & 1000 & 2670 & 1090 & 2200 & 1501 & & 1000 & 4810 & 2195 & 2500 \\
\hline
\end{tabular}

$\beta$ are the compressional and shear wave velocities $\left(\mathrm{ms}^{-1}\right)$, respectively, and $\rho$ is the material density $\left(\mathrm{kgm}^{-3}\right)$. Materials with $\alpha=2670 \mathrm{~ms}^{-1}$ correspond to sandstone, while those with $\alpha=$ $4810 \mathrm{~ms}^{-1}$ correspond to limestone. Results are described in the following paragraphs.

Figure 3 presents the displacement spectra, for the three interfaces models studied, for the first receiver detailed in Figure 1. The depth to which the source is applied is $H=$ $0.05 \mathrm{~m}$ and the horizontal distance from the source to the receiver is $20 \mathrm{H}=1.0 \mathrm{~m}$. The response corresponding to Materials 1 is shown in Figures 3(a) and 3(b), while that associated with the Materials 2 are graphed in Figures 3(c) and 3(d). For the analysis, a frequency increment of $150 \mathrm{~Hz}$ was considered and a maximum frequency of $19200 \mathrm{~Hz}$ was reached.

Results associated with Materials 1 show clear amplifications for vacuum-solid and water-solid interfaces for both directions of displacement. At low frequencies, these two interfaces describe similar amplitudes (for frequencies lower than $1000 \mathrm{~Hz}$ ). For frequencies close to $19000 \mathrm{~Hz}$, the behavior becomes asymptotic and almost negligible. For the model formed by water and sandstone, strong variations of displacement are noted for the range of 1000 to $12000 \mathrm{~Hz}$, mainly for the $x_{3}$ component. As was expected, the model formed by the $S^{1}$ and $S^{2}$ shows amplitudes of displacement that are much smaller, and its behavior describes soft patterns. From the frequency of $10000 \mathrm{~Hz}$, the behavior is almost negligible in both directions.

The response obtained from Materials 2 for the three models is depicted in Figures $3(\mathrm{c})$ and $3(\mathrm{~d})$. It is possible to appreciate that for both directions of displacement, the spectra show soft trajectories and almost negligible from the frequency of $8000 \mathrm{~Hz}$. This behavior can be attributed to the great rigidity of the bottom material (limestone) in comparison with the top material (air, wáter, or sandstone). This stiffness not only has dominion or control in the response (displacements), but also, provides a certain similarity in the spectra, and therefore, on the Rayleigh's, Stoneley's and Scholte's interface waves for these materials.

Figure 4 shows synthetic seismograms of displacement for the directions $x_{1}$ and $x_{3}$ (left and right, resp.), measured by 25 receivers located as depicted in Figure 1. The first receiver is located at a horizontal distance of $20 \mathrm{H}=1.0 \mathrm{~m}$ from the source. The other receivers are located using a distance increment of $0.04 \mathrm{~m}$. For each of the interface models studied is evident the emergence of their corresponding interface waves, that is, Rayleigh's, Stoneley's, and Scholte's waves, for vacuum-solid, solid-solid, and water-solid interfaces, Figures 4(a), $4(\mathrm{~b})$, and 4(c), respectively.

Figure 4(a) shows the arrival of $P$ waves at a speed of approximately $2670 \mathrm{~ms}^{-1}$; also the arrival of Rayleigh's waves traveling close to $1021 \mathrm{~ms}^{-1}$ is observed. Here, the amount of energy carrying Rayleigh's waves is clear. For the case of two-solid interface, limestone (top) and sandstone (bottom), the emergence of Stoneley's waves is expected. In Figure 4(b), it is possible to look at three wave fronts, which propagate at speeds of $4810 \mathrm{~ms}^{-1}, 1.850 \mathrm{~ms}^{-1}$, and $1078 \mathrm{~ms}^{-1}$. The first wave front is associated with the speed of compressional waves of the 

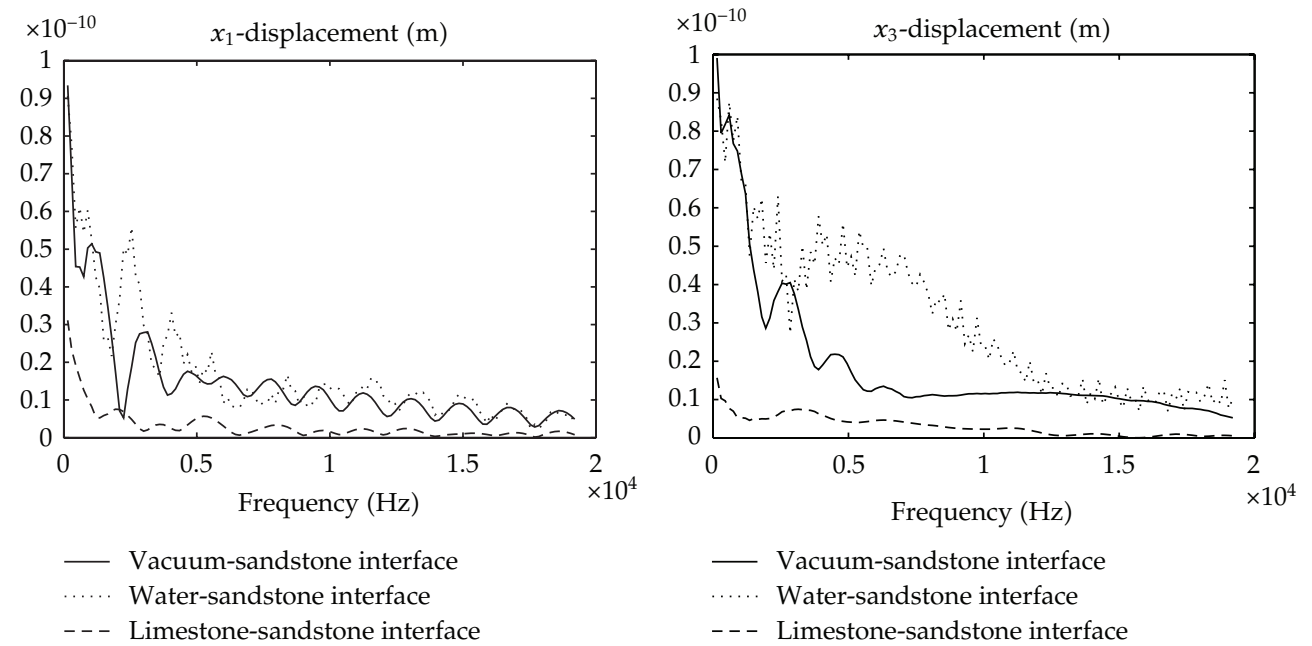

(b)
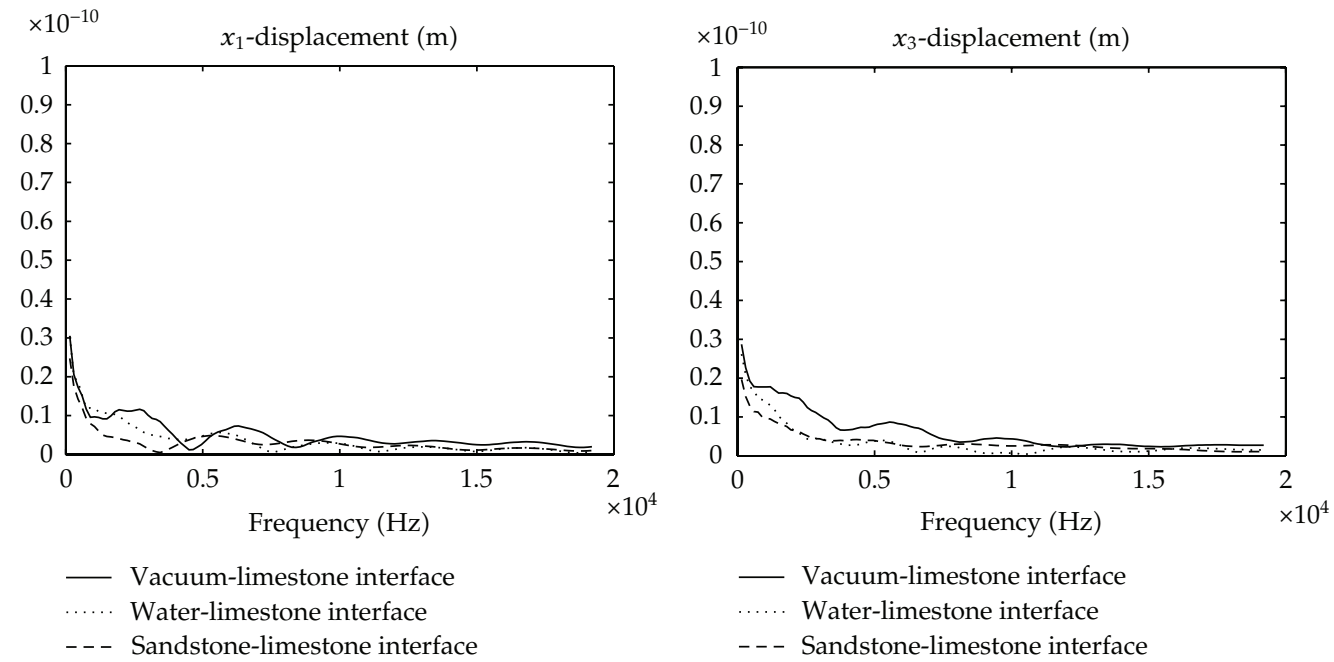

(d)

Figure 3: Spectra of displacement for three interface models. Results for Materials 1 (see Table 1) are plotted (a) and (b), while those obtained from Materials 2 are presented in (c) and (d).

limestone, the second is close to the shear waves velocity of limestone, and the last shows a velocity of less than the shear wave velocity of sandstone and can be associated with the propagation of Stoneley's waves. In Figure 4(c) two wave fronts are highlighted. The first traveling at a velocity of $2670 \mathrm{~ms}^{-1}$ and is associated with the compressional wave velocity of sandstone. The second front corresponds to the propagation of Scholte's waves whose speed is $970 \mathrm{~ms}^{-1}$ and obviously carries a significant amount of energy, mainly in its $x_{3}$ component.

In the case of interface waves for Materials 2 of Table 1, it should be emphasized that results for this material describes similar behavior to Materials 1. In general, the amplitudes of displacement, for both components $x_{1}$ and $x_{3}$, are lower than those obtained for Materials 1. This is due to the great rigidity of limestone compared with sandstone. In the synthetic seismograms presented in Figure 5, different wave front arrivals and their corresponding speeds 

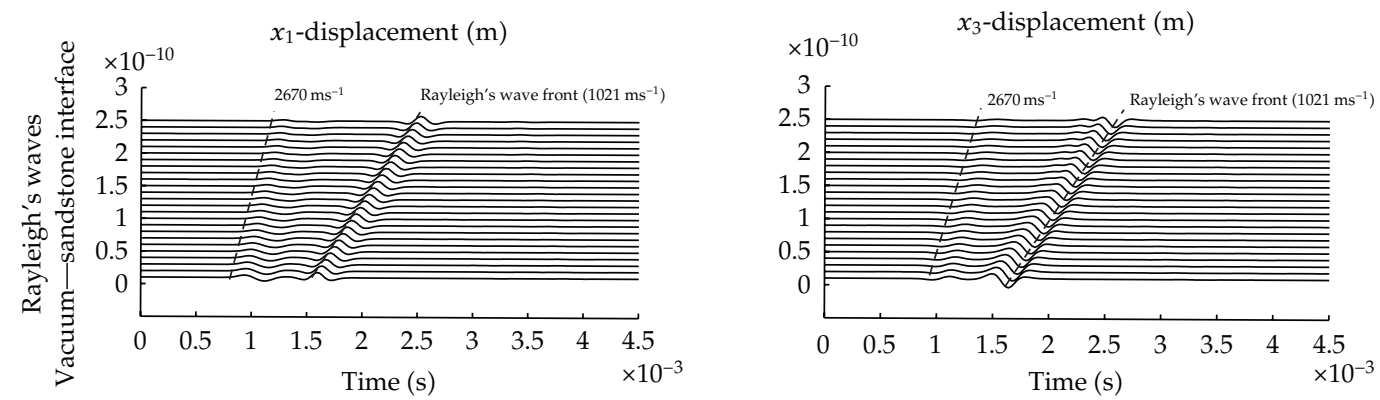

(a)
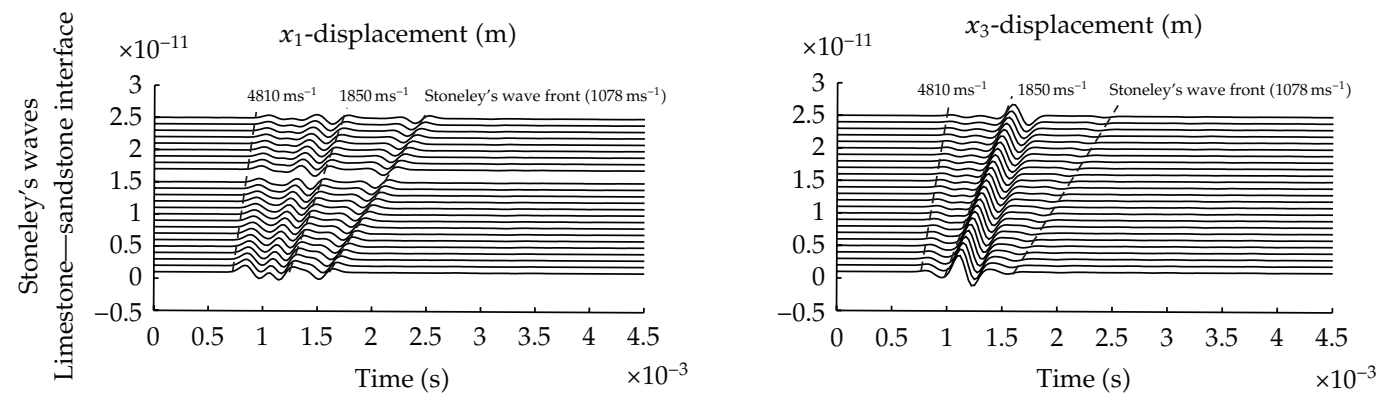

(b)
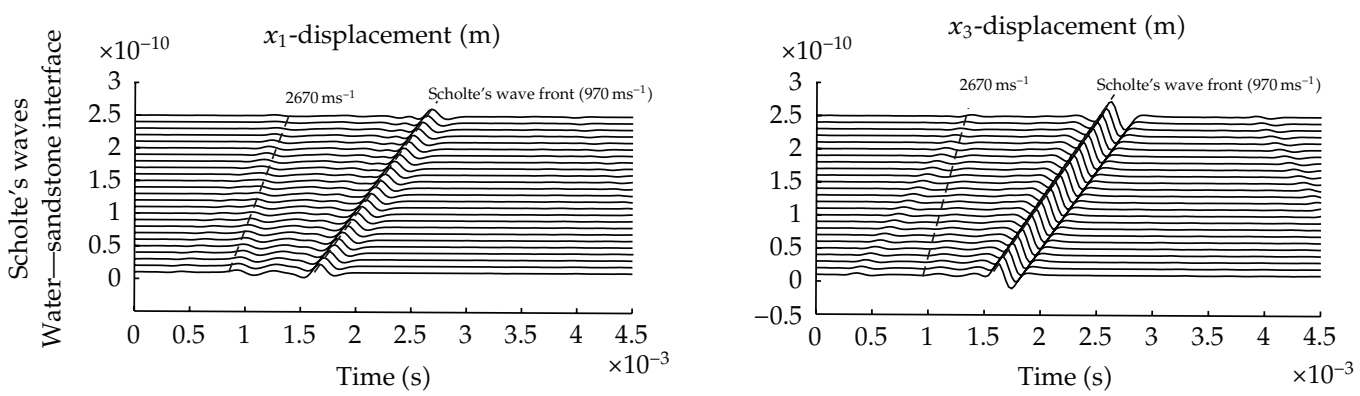

(c)

Figure 4: Synthetic seismograms for three different interfaces. (a) Vacuum-solid interface; (b) solid-solid interface, and (c) fluid-solid interface. $x_{1}$ (left) and $x_{3}$ (right) components of displacement are plotted. Materials 1 from the Table 1 are used for the analysis.

are highlighted. It can be seen the amount of energy that leads the different waves that propagate in the interface, manifesting itself in the amplitudes of the displacement generated. In Figure 3(c), it is important to mention the influence of fluid layer, showing a wave front that propagates at a speed of $1501 \mathrm{~ms}^{-1}$.

Figures $6(\mathrm{a})$ and $6(\mathrm{~b})$ show the time response for the entire $2 \mathrm{D}$ water-sandstone interface model. To this purpose, a net of $51 \times 51$ receivers, spaced using a distance increment of $0.04 \mathrm{~m}$, is required. Column (a) plots the results of pressure in the fluid and displacements in the $x_{1}$ direction for the solid, while the column (b) plots pressures in the fluid and displacements in the $x_{3}$ direction for the solid. This numerical simulation is shown for three different times. 

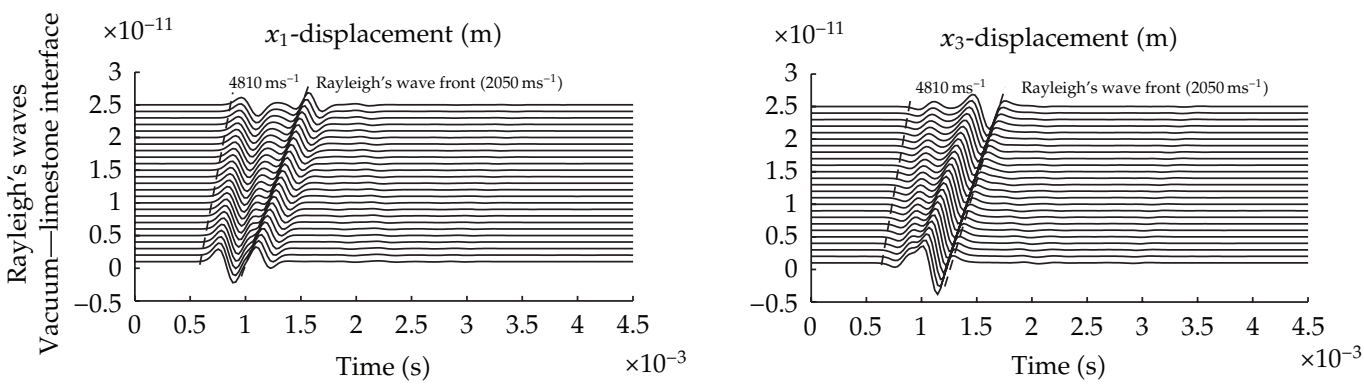

(a)
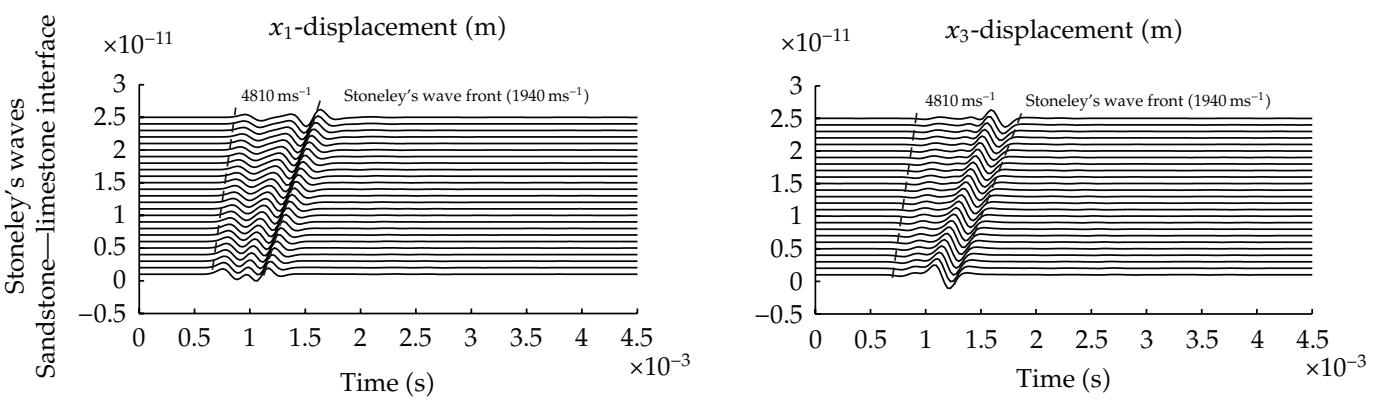

(b)
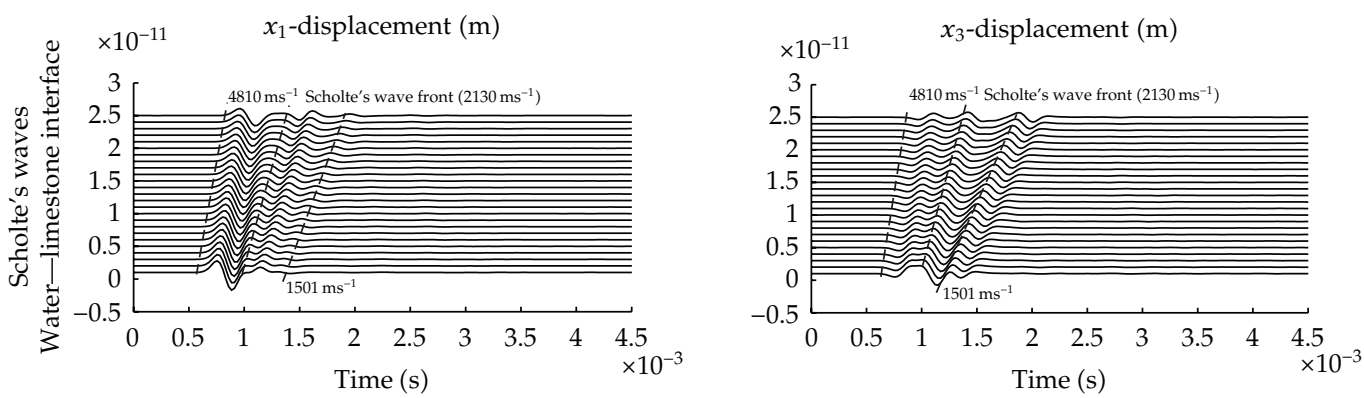

(c)

Figure 5: Synthetic seismograms for three different interfaces. (a) Vacuum-solid interface, (b) solid-solid interface, and (c) fluid-solid interface. $x_{1}$ (left) and $x_{3}$ (right) components of displacement are plotted. Materials 2 from the Table 1 are used for the analysis.

For the time $t=0.000911 \mathrm{~s}$, the source has hit the solid boundary and a diffracted wave in the fluid and reflected waves in the solid can be seen, generating the emergence of $P$ y $S$ wave fronts. For the time $t=0.001432 \mathrm{~s}$, the above mentioned waves go away from the source while the presence of interface waves is clearly evident to this time. These are the Scholte's waves and are highlighted using circles in Figures 6(a) and 6(b). For the time $t=0.001953 \mathrm{~s}$, the propagation of interface waves is very visibly and shows a delay with respect to the $P$ and $S$ wave fronts in the solid. Scholte's waves for this case propagate with a velocity of $970 \mathrm{~ms}^{-1}$.

\section{Conclusions}

In this work, we expand the use of the indirect boundary element method to study the propagation of elastic waves in vacuum-solid, solid-solid, and fluid-solid interfaces. In this 

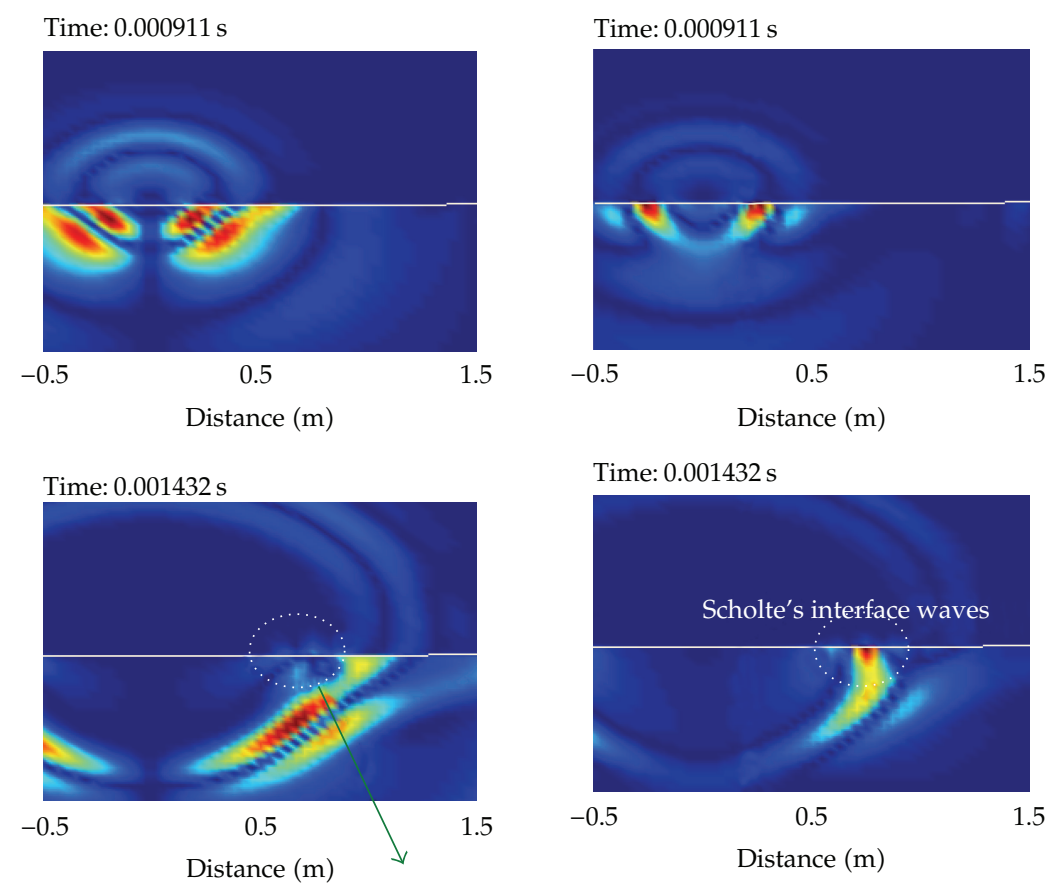

Time: $0.001432 \mathrm{~s}$

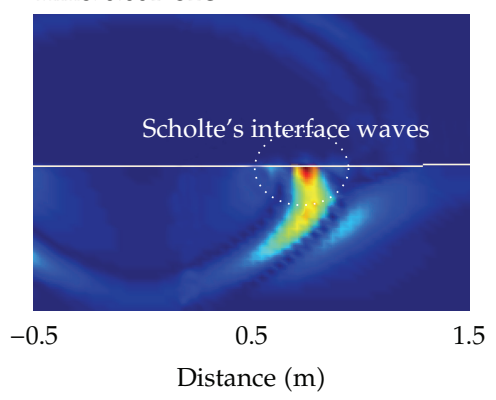

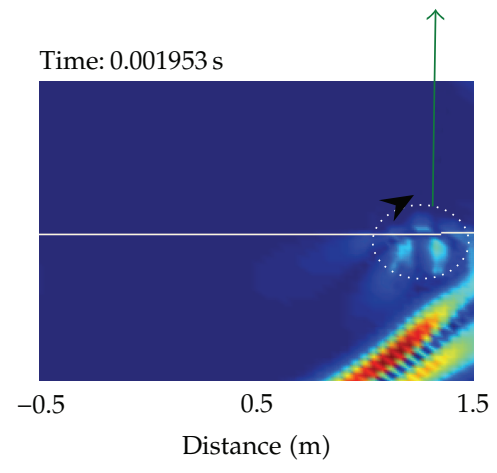

(a)

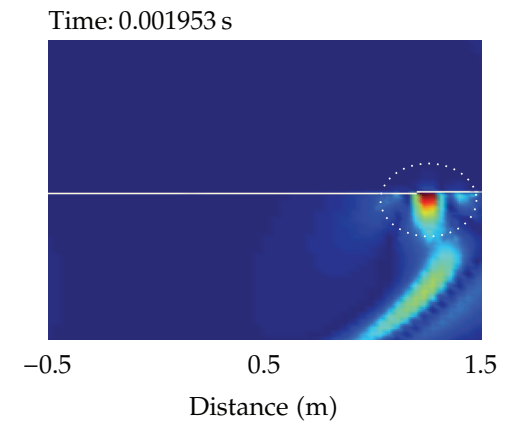

(b)

Figure 6: Snapshots by IBEM for the complete 2D water-sandstone interface model are shown. A grid of $51 \times 51$ receivers, spaced using a distance increment of $0.04 \mathrm{~m}$, is required. Column (a) plots the results of pressures in the fluid and displacements in the $x_{1}$ direction for the solid, while the column (b) plots pressures in the fluid and displacements in the $x_{3}$ direction for the solid.

numerical technique, based on Huygens' principle and the Somigliana's representation theorem, the fields of pressures, tractions, and displacements are expressed in terms of singlelayer boundary integral equations.

Green's functions for tractions and displacements, for unbounded space, were used, but they are enforced to meet the proper boundary conditions that prevail for each interface model studied.

Firstly, spectra of displacements were included, and some aspects about the behavior for the two groups of materials studied were pointed out. Therefore, a fast fourier transform 
algorithm was applied to obtain time responses for the three interface models. In all cases, the existence and propagation of Rayleigh, Stoneley, and Scholte's waves are manifested, highlighting the important amount of energy that they transport.

The results obtained from our numerical technique were compared with the DWN; a good agreement between the different approaches was evident. Therefore, the IBEM can be considered as a good technique to model interface problems. Complex geometries solved by means of IBEM are the target of our current research.

\section{Appendix}

This section presents the validation of the indirect boundary element method for the solution of interface problems and compares results by IBEM with those obtained by the discrete wave number. For this purpose, a fluid-solid interface has been selected. In this case, a source is applied in the fluid, which is represented by a Hankel's function of second kind and zero order. The following is a brief description of the DWN method [35].

The discrete wave number method is one of the techniques to simulate earthquake ground motions. The seismic wave radiated from a source is expressed as a wavenumber integration [29]. The main idea of the method is to represent a source as a superposition of homogenous plane waves propagating in discrete angles. As long as the medium has no inelastic damping, the denominator of the integrand becomes zero for a particular wavenumber and, consequently, the numerical integration becomes impossible. To solve this problem, a method to incorporate a complex frequency was proposed as early as the proposal of the discrete wavenumber method itself.

The incident pulse at the fluid (source) can be expressed as

$$
p^{0^{F}}(\mathbf{x})=C(\omega) H_{0}^{(2)}\left(\frac{\omega r}{c^{F}}\right)=\frac{C(\omega)}{\pi} \int_{-\infty}^{\infty} \frac{e^{-i k x_{3}-i \eta\left|x_{1}\right|}}{\eta} d k \approx \frac{C(\omega)}{\pi} \sum_{n=-N}^{N} \frac{e^{-i k_{n} x_{3}-i \eta_{n}\left|x_{1}\right|}}{\eta_{n}} \Delta k
$$

where $p^{0^{F}}(\mathbf{x})$ is the incident pulse at the fluid, $\mathbf{x}=\left\{x_{1}, x_{3}\right\}, C(\omega)$ represents a scale factor for the incident pulse, $H_{0}^{(2)}(\cdot)$ is Hankel's function of second kind and zero order, $\omega$ is circular frequency, $c^{F}$ represents the compressional wave velocity for the fluid, and $r=r(\mathbf{x})$ is the distance from the receiver to the source. $k$ is the wavenumber, $\eta=\sqrt{\left(\omega^{2} / c^{F^{2}}\right)-k^{2}}$ with $\operatorname{Im} \eta<0$. If we express $k$ in discrete values, then we have $k_{n}=n \Delta k$ and $\eta_{n}=\sqrt{\left(\omega^{2} / c^{F^{2}}\right)-k_{n}^{2}}$ with $\operatorname{Im} \eta_{n}<0$.

If we assume that the whole pressure and displacement field in the fluid, that is, free and diffracted field, can be expressed, respectively, by

$$
\begin{gathered}
p^{F}(\mathbf{x})=p^{0^{F}}(\mathbf{x})+p^{d^{F}}(\mathbf{x})=p^{0^{F}}(\mathbf{x})+\sum_{n=-N}^{N} A_{n} e^{-i k_{n} x_{3}+i \eta_{n}\left(x_{1}-a\right)}, \\
u_{1}^{F}(\mathbf{x})=\frac{1}{\rho \omega^{2}} \frac{\partial p^{F}(\mathbf{x})}{\partial x_{1}}=\frac{1}{\rho \omega^{2}}\left\{\sum_{n=-N}^{N} \frac{-i \operatorname{sig}\left(x_{1}\right)}{\pi} e^{-i k_{n} x_{3}-i \eta_{n}\left|x_{1}\right|} \Delta k+\sum_{n=-N}^{N} i A_{n} \eta_{n} e^{-i k_{n} x_{3}+i \eta_{n}\left|x_{1}-a\right|} \Delta k\right\} .
\end{gathered}
$$




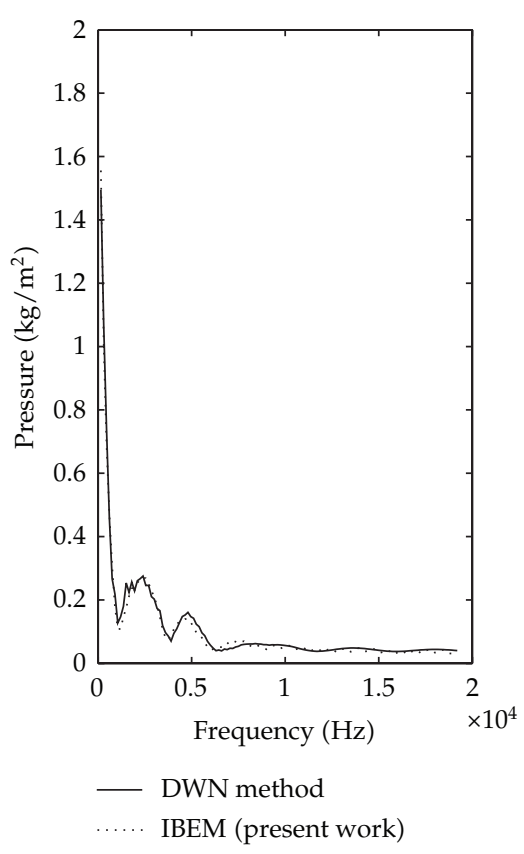

(a)

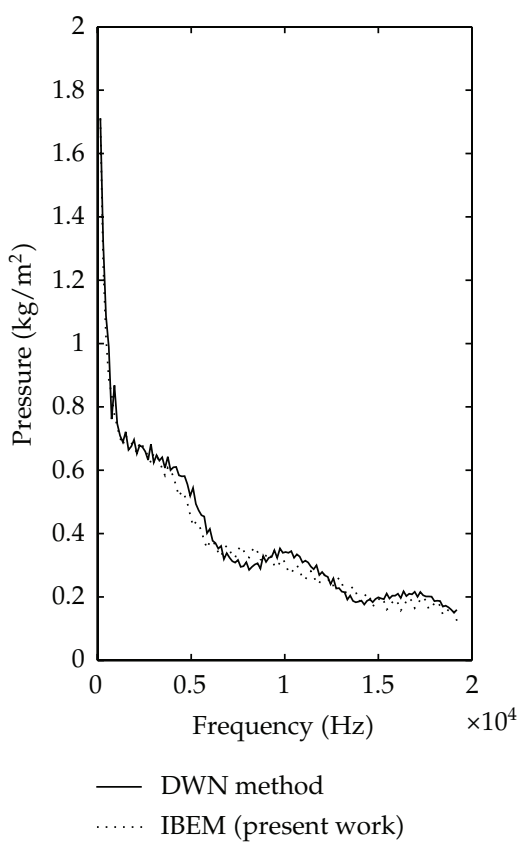

(b)

Figure 7: Spectra of pressures for water-sandstone (left) and water-limestone (right) interfaces. Results obtained by IBEM are plotted using dotted lines, while those obtained by means of DWN are drawn with continuous lines. Good agreement between IBEM and DWN is observed.

$A_{n}$ represents the unknown coefficient to evaluate the diffracted field in the fluid and $a$ is the distance from the source to the elastic solid.

For the solid, we assume that the potential of displacement has the form $\phi=$ $\sum B_{n} e^{-i k_{n} x_{3}} e^{-i \gamma_{n}\left(x_{1}-a\right)}$ and $\psi=\sum C_{n} e^{-i k_{n} x_{3}} e^{-i v_{n}\left(x_{1}-a\right)}$, where $\gamma_{n}=\sqrt{\left(\omega^{2} / \alpha^{2}\right)-k_{n}^{2}}$ with $\operatorname{Im} \gamma_{n}\langle 0$ and $v_{n}=\sqrt{\left(\omega^{2} / \beta^{2}\right)-k_{n}^{2}}$ with $\operatorname{Im} v_{n}\langle 0 . \alpha$ and $\beta$ are the compressional and shear wave velocities, respectively.

The displacement field for the solid can be expressed as $u=\partial \phi / \partial x_{1}-\partial \psi / \partial x_{3}$ and $w=\partial \phi / \partial x_{3}+\partial \psi / \partial x_{1}$. The stress field can be obtained by the well-known equation:

$$
\sigma_{i j}(\mathbf{x})=\lambda \varepsilon_{k k} \delta_{i j}+2 \mu \varepsilon_{i j}
$$

where $\sigma_{i j}(\mathbf{x})$ is stress tensor, $\lambda$ and $\mu$ are Lamé's constants, $\varepsilon_{i j}$ is strain tensor and $\delta_{i j}$ is Kronecker's delta.

The boundary conditions to be enforced are represented by (3.6). Once the boundary conditions have been applied, the unknown coefficients $A_{n}, B_{n}$, and $C_{n}$ are obtained, the whole pressure field in the fluid is finally determined by means of (A.2).

For validation purposes, water-sandstone and water-limestone interfaces are considered (for material properties see Table 1). Figure 7 shows the spectra of pressures for the models analyzed. For all cases, the initial pressure (source) was generated in the fluid at a distance of $0.05 \mathrm{~m}$ from the elastic solid boundary. The receiver is placed at a horizontal distance of $1.0 \mathrm{~m}$ from the source. The frequency analysis is done considering a frequency increment of 
$150 \mathrm{~Hz}$ and reaching a maximum of $19200 \mathrm{~Hz}$. In Figure 7, results from IBEM are plotted with dotted line, while those obtained by DWN are drawn with continuous line. It can be seen that both techniques coincide acceptably.

\section{Acknowledgment}

Thanks are given to the SENER-CONACYT Project 128376 and to the Instituto Mexicano del Petróleo.

\section{References}

[1] J. W. S. Rayleigh, "On waves propagated along the plane surface of an elastic solid," Proceedings London Mathematical Society, vol. 17, no. 1, pp. 4-11, 1985.

[2] H. L. Wong and P. C. Jennings, "Effects of canyon topography on strong ground motion," Bulletin of the Seismological Society of America, vol. 65, no. 5, pp. 1239-1257, 1975.

[3] H. L. Wong, "Effect of surface topography on the diffraction of P, SV and Rayleigh waves," Bulletin of the Seismological Society of America, vol. 72, no. 4, pp. 1167-1183, 1982.

[4] F. J. Sànchez-Sesma and M. Campillo, "Diffraction of P, SV and Rayleigh waves by topographic features: a boundary integral formulation," Bulletin of Seismological Society of America, vol. 81, no. 6, pp. 2234-2253, 1991.

[5] A. Rodríguez-Castellanos, F. Luzón, and F. J. Sánchez-Sesma, "Diffraction of seismic waves in an elastic, cracked halfplane using a boundary integral formulation," Soil Dynamics and Earthquake Engineering, vol. 25, no. 11, pp. 827-837, 2005.

[6] R. Stoneley, "Elastic waves at the surface of separation between two solids," Proceedings of the Royal Society of London. Series A, vol. 106, no. 738, pp. 416-428, 1924.

[7] D. Rauch, "On the role of bottom interface waves in ocean seismo-acoustics: a review," Marine Science, vol. 16, pp. 623-641, 1986.

[8] G. D. Meegan, M. F. Hamilton, Y. A. Il'inskii, and E. A. Zabolotskaya, "Nonlinear Stoneley and Scholte waves," Journal of the Acoustical Society of America, vol. 106, no. 4, pp. 1712-1723, 1999.

[9] N. F. Declercq, J. Degrieck, R. Briers, and O. Leroy, "Diffraction of homogeneous and inhomogeneous plane waves on a doubly corrugated liquid/solid interface," Ultrasonics, vol. 43, no. 8, pp. 605-618, 2005.

[10] A. P. Kiselev and D. F. Parker, “Omni-directional Rayleigh, Stoneley and Schölte waves with general time dependence," Proceedings of The Royal Society of London. Series A. Mathematical, Physical and Engineering Sciences, vol. 466, no. 2120, pp. 2241-2258, 2010.

[11] J. G. Scholte, “On the Stoneley wave equation," Proceedings of the Koninklijke Nederlandse Akademie van Wetenschappen, vol. 45, part 1, pp. 20-25, 1942.

[12] J. G. Scholte, "On the Stoneley wave equation," Proceedings of the Koninklijke Nederlandse Akademie van Wetenschappen, vol. 45, part 2, pp. 159-164, 1942.

[13] J. G. Scholte, "The range of existence of Rayleigh and Stoneley waves," Royal Astronomical Society. Geophysics, supplement 5, pp. 120-126, 1947.

[14] M. A. Biot, "The interaction of Rayleigh and Stoneley waves in the ocean bottom," Bulletin of the Seismological Society of America, vol. 42, pp. 81-93, 1952.

[15] W. M. Ewing, W. S. Jardetzky, and F. Press, Elastic Waves in Layered Media, McGraw-Hill Book Companies, New York, NY, USA, 1957.

[16] M. Yoshida, "Velocity and response of higher mode Rayleigh waves for the Pacific Ocean," Bulletin of the Earthquake Research Institute, vol. 53, pp. 1135-1150, 1978.

[17] M. Yoshida, "Group velocity distributions of Rayleigh waves and two upper mantle models in the Pacific Ocean," Bulletin of the Earthquake Research Institute, vol. 53, pp. 319-338, 1978.

[18] O. C. Zienkiewicz and P. Bettess, "Fluid-structure dynamic interaction and wave forces, an introduction to numerical treatment," International Journal for Numerical Methods in Engineering, vol. 13, no. 1, pp. 1-16, 1978.

[19] R. van Vossen, J. O. A. Robertsson, and C. H. Chapman, "Finite-difference modeling of wave propagation in a fluid-solid configuration," Geophysics, vol. 67, no. 2, pp. 618-624, 2002. 
[20] A. Rodríguez-Castellanos, E. F. Mendez, F. J. Sánchez-Sesma, and J. E. Rodríguez-Sánchez, “Numerical formulation to study fluid-solid interfaces excited by elastic waves," Key Engineering Materials, vol. 449, pp. 54-61, 2010.

[21] A. Rodríguez-Castellanos, E. Flores, F. J. Sánchez-Sesma, C. Ortiz-Alemán, M. Nava-Flores, and R. Martin, "Indirect boundary element method applied to fluid-solid interfaces," Soil Dynamics and Earthquake Engineering, vol. 31, no. 3, pp. 470-477, 2011.

[22] D. Komatitsch, C. Barnes, and J. Tromp, "Wave propagation near a fluid-solid interface: a spectralelement approach," Geophysics, vol. 65, no. 2, pp. 623-631, 2000.

[23] J. M. Carcione and H. B. Helle, "The physics and simulation of wave propagation at the ocean bottom," Geophysics, vol. 69, no. 3, pp. 825-839, 2004.

[24] J. M. Carcione, B. H. Helle, G. Seriani, and M. P. Plasencia Linares, "Simulation of seismograms in a 2-D viscoelastic earth by pseudospectral methods," Geofisica Internacional, vol. 44, no. 2, pp. 123-142, 2005.

[25] L. Jian-Fei and J. Dong-Sheng, "Dynamic response of an offshore pile to pseudo-Stoneley waves along the interface between a poroelastic seabed and seawater," Soil Dynamics and Earthquake Engineering, vol. 30, no. 4, pp. 184-201, 2010.

[26] M. Bouchon, "A numerical simulation of the acoustic and elastic wavefields radiated by a source on a fluid-filled borehole embedded in a layered medium," Geophysics, vol. 58, no. 4, pp. 475-481, 1993.

[27] G. Havin, O. Abraham, H. A. Pedersen, and M. Campillo, "Characterisation of surface cracks with rayleigh waves: a numerical model," NDT and E International, vol. 31, no. 4, pp. 289-297, 1998.

[28] W. Liu, Y. Cho, H. Phan, and J. D. Achenbach, "Study on the scattering of 2-D Rayleigh waves by a cavity based on BEM simulation," Journal of Mechanical Science and Technology, vol. 25, no. 3, pp. 797-802, 2011.

[29] Z. L. Li and J. D. Achenbach, "Reflection and transmission of Rayleigh surface waves by a material interphase," Journal of Applied Mechanics, Transactions ASME, vol. 58, no. 3, pp. 688-694, 1991.

[30] S. Hirose, Y. Ushida, and C. Y. Wang, "BEM analysis of wave propagation in a water-filled borehole in an anisotropic solid," Tsinghua Science and Technology, vol. 12, no. 5, pp. 520-526, 2007.

[31] A. Rodríguez-Castellanos, F. J. Sánchez-Sesma, F. Luzón, and R. Martin, "Multiple scattering of elastic waves by subsurface fractures and cavities," Bulletin of the Seismological Society of America, vol. 96, no. 4, pp. 1359-1374, 2006.

[32] E. Strick and A. S. Ginzbarg, "Stoneley-wave velocities for a fluid-solid interface," Bulletin of the Seismological Society of America, vol. 46, no. 4, pp. 281-292, 1956.

[33] E. Strick, W. L. Roever, and T. F. Vining, "Theoretical and experimental investigation of a PseudoRayleigh wave," Journal of the Acoustical Society of America, vol. 28, no. 4, pp. 794-794, 1956.

[34] P. Borejko, "A new benchmark solution for the problem of water-covered geophysical bottom," in Proceedings of the International Symposium on Mechanical Waves in Solids, Zhejiang University, Hangzhou, China, 2006.

[35] M. Bouchon and K. Aki, "Discrete wave number representation of seismic source wave fields," Bulletin of the Seismological Society of America, vol. 67, no. 2, pp. 259-277, 1977. 


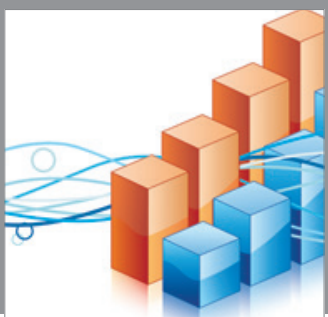

Advances in

Operations Research

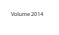

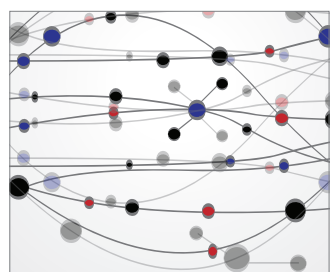

\section{The Scientific} World Journal
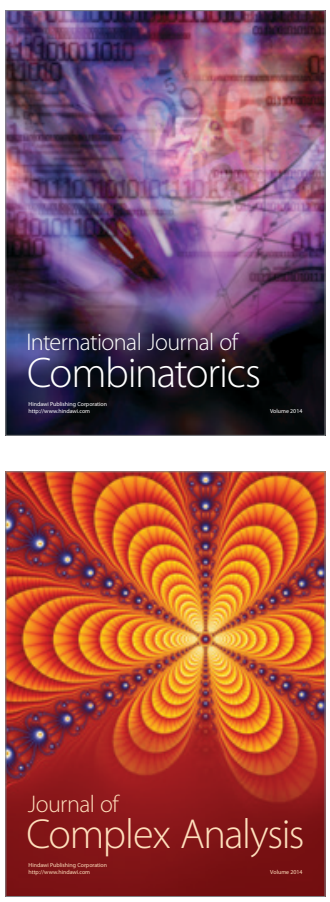

International Journal of

Mathematics and

Mathematical

Sciences
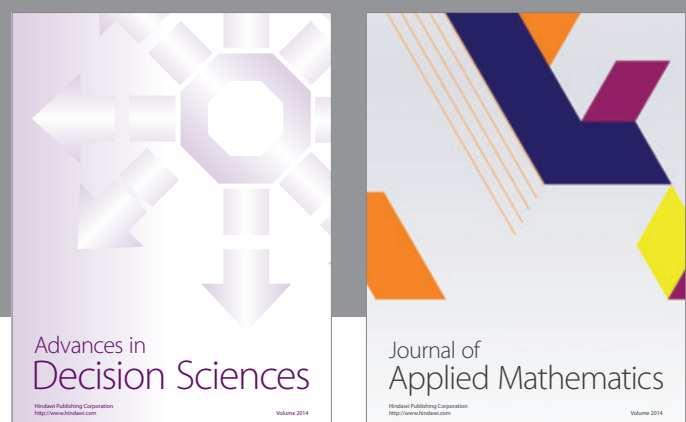

Journal of

Applied Mathematics
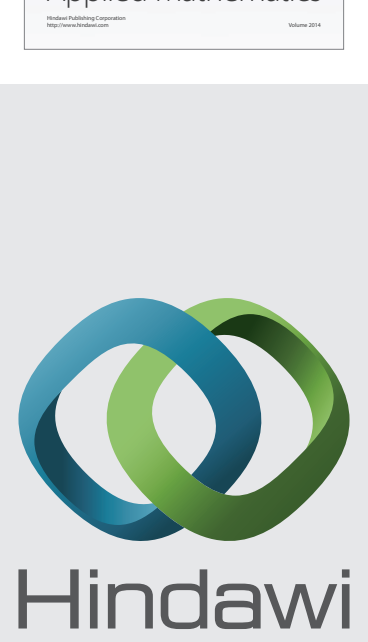

Submit your manuscripts at http://www.hindawi.com
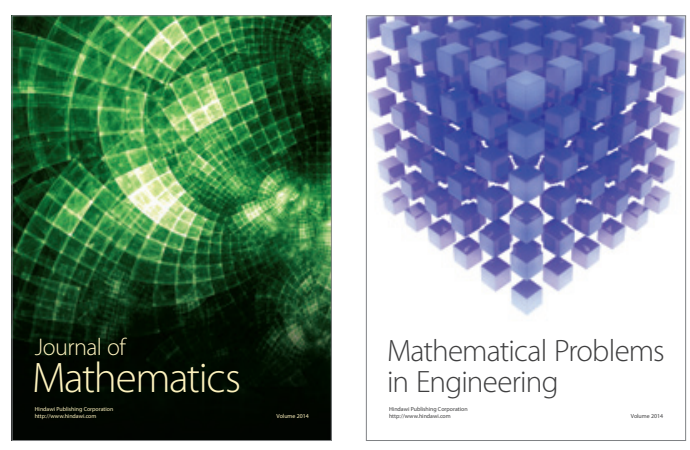

Mathematical Problems in Engineering
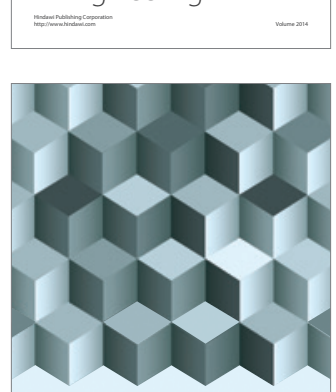

Journal of

Function Spaces
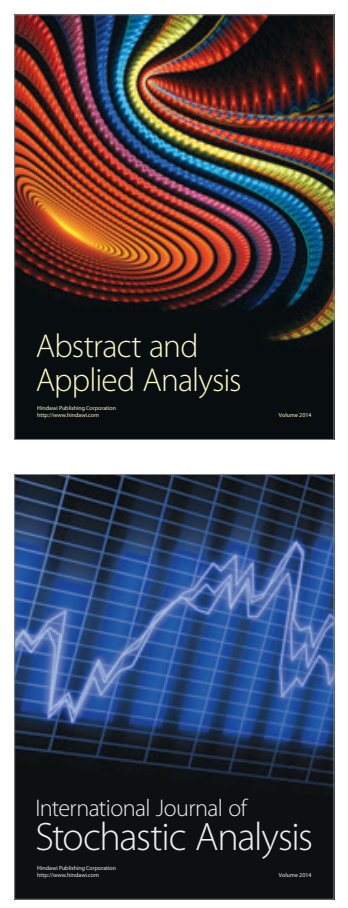

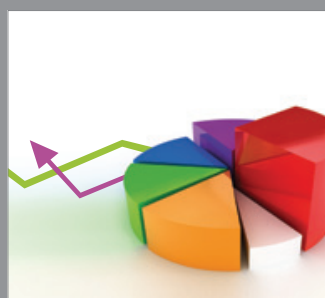

ournal of

Probability and Statistics

Promensencen
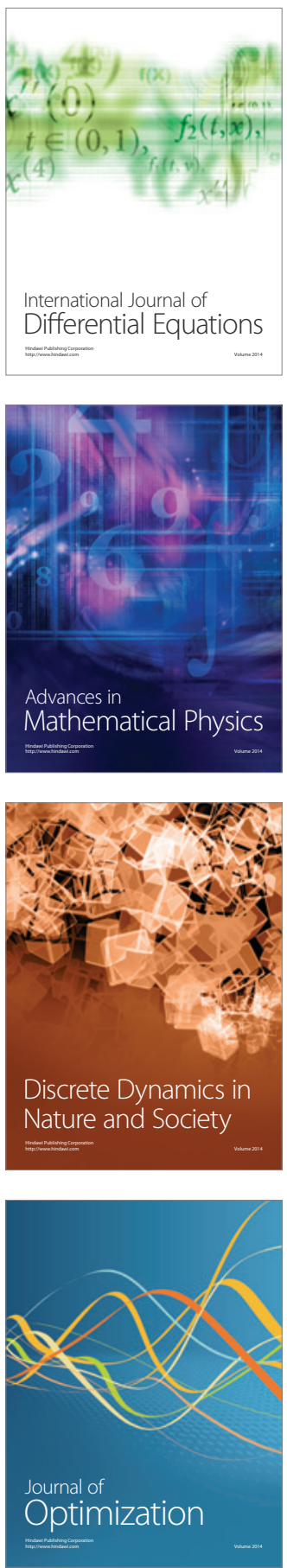